\title{
Factorial Characters and Tokuyama's Identity for Classical Groups
}

\author{
Angèle M. Hamel $\|^{\|}$and Ronald C. King非 \\ ${ }^{1}$ Dept. of Physics and Computer Science, Wilfrid Laurier University, Waterloo, Ontario, Canada \\ ${ }^{2}$ School of Mathematical Sciences, University of Southampton, Southampton, United Kingdom
}

\begin{abstract}
In this paper we introduce factorial characters for the classical groups and derive a number of central results. Classically, the factorial Schur function plays a fundamental role in traditional symmetric function theory and also in Schubert polynomial theory. Here we develop a parallel theory for the classical groups, offering combinatorial definitions of the factorial characters for the symplectic and orthogonal groups, and further establish flagged factorial Jacobi-Trudi identities and factorial Tokuyama identities, providing proofs in the symplectic case. These identities are established by manipulating determinants through the use of certain recurrence relations and by using lattice paths.

Résumé. Ici nous présentons des caractères factoriels pour des groupes classiques, et nous dérivons plusieurs résultats importants. Classiquement, le fonction de Schur factoriel joue une rôle fondamentale dans la théorie traditionnelle des fonctions symétriques et aussi dans la théorie des polynômes de Schubert. Ici nous développons une théorie paralléle à celle des groupes classiques, offrons des définitions combinatoire des caractères factoriels pour des groupes symplectiques et orthogonaux, et nous établissons aussi des identités de «flagged factorial Jacobi Trudi » et de «factorial Tokyama », donnant des preuves dans le cas symplectique. Ces identités sont etablies par la manipulation des déterminants en utilisant de certains récurrences, et en utliisant des chemins.
\end{abstract}

Keywords. factorial symmetric functions, Jacobi-Trudi identity, Tokuyama identity, classical Lie groups

\section{Introduction}

Factorial Schur functions-introduced by Biedenharn and Louck [1], extended by Goulden and Greene [6] and Macdonald [12] — have a fundamental role to play in symmetric function theory, and are intimately connected to Schubert calculus through the double Schubert polynomials. As such, they have been the object of much active research, and numerous results and applications have been established for them [9]. However, what are the factorial characters for other classical Lie groups? Given the character interpretations - as ratios of determinants - can we derive the fundamental theorems of this type for them: Jacobi-Trudi and Tokuyama identities? These are the questions we consider in this paper.

Classical group analogues of factorial Schur functions have been studied previously in the context of double Schubert polynomials. Notably, Ikeda et al. [9] have made use of the factorial Schur P and Q

\footnotetext{
${ }^{\dagger}$ Email: ahamel@wlu.ca.

‡Email: r.c.king@soton.ac.uk

1365-8050 (c) 2016 Discrete Mathematics and Theoretical Computer Science (DMTCS), Nancy, France
} 
functions (see also Ivanov [10]) to construct double Schubert polynomials in the case of classical Lie groups (building on Billey and Haiman [2]) To the best of our knowledge we are the first to offer explicit definitions of factorial characters themselves for the symplectic and odd orthogonal groups. Furthermore, on the basis of these definitions, we derive both flagged Jacobi-Trudi and Tokuyama identities.

In the non-factorial case the flagged Jacobi-Trudi identities for the symplectic group appears in [5] and for all classical groups in [14]. Currently one of the most general forms of Tokuyama's original identity [19] appears in Theorem 1 below, where the definitions of $Q_{\lambda}$ and $s_{\mu}$ follow in a later section. A brief table summarizes the history of the results.

Theorem 1 Let $\mu$ be a partition of length $\ell(\mu) \leq n$ and $\lambda=\mu+\delta$ with $\delta=(n, n-1, \ldots, 1)$. Then for $x=\left(x_{1}, x_{2}, \ldots, x_{n}\right), y=\left(y_{1}, y_{2}, \ldots, y_{n}\right)$, and $a=\left(a_{1}, a_{2}, \ldots\right)$.

$$
Q_{\lambda}(\mathbf{x} ; \mathbf{y} \mid \mathbf{a})=\prod_{1 \leq i \leq j \leq n}\left(x_{i}+y_{j}\right) s_{\mu}(\mathbf{x} \mid \mathbf{a}) .
$$

\begin{tabular}{|l|l|l|l|}
\hline Tokuyama [19],1988 & $\mathbf{x}$ & $\mathbf{y}=t \mathbf{x}$ & $\mathbf{a}=0$ \\
Okada [13], 1990 & $\mathbf{x}$ & $\mathbf{y}=t \mathbf{x}$ & $\mathbf{a}=0$ \\
Hamel and King [7], 2007 & $\mathbf{x}$ & $\mathbf{y}$ & $\mathbf{a}=0$ \\
Brubaker, Bump, Friedberg [3], 2011 & $\mathbf{x}$ & $\mathbf{y}$ & $\mathbf{a}=0$ \\
Ikeda, Mihalcea, Naruse [9], 2011 & $\mathbf{x}$ & $\mathbf{y}=\mathbf{x}$ & $\mathbf{a}$ \\
Bump, McNamara, Nakasuji [4], 2011 & $\mathbf{x}$ & $\mathbf{y}=t \mathbf{x}$ & $\mathbf{a}$ \\
Hamel and King [8], 2015 & $\mathbf{x}$ & $\mathbf{y}$ & $\mathbf{a}$ \\
\hline
\end{tabular}

Our new factorial characters are defined in Section 2 . Section 3 gives lemmas leading to the flagged factorial Jacob-Trudi identities (Theorem 6). In Section 4 we give explicit formulae appropriate to the one-part partition case, and use them in Section 5 to provide combinatorial expressions for our factorial characters in terms of lattice paths and then tableaux (Theorem 11) in an approach similar to that of Ivanov's appendix to [15]. In Section 6, on the basis of new definitions of factorial $Q$-functions, we derive factorial Tokuyama type identities (Theorem 17).

\section{Factorial characters for $G L(n, \mathbb{C}), S p(2 n, \mathbb{C})$ and $S O(2 n+1, \mathbb{C})$}

Let $n \in \mathbb{N}$ be fixed. Let $\mathbf{x}=\left(x_{1}, x_{2}, \ldots, x_{n}\right)$ and $\overline{\mathbf{x}}=\left(\bar{x}_{1}, \bar{x}_{2}, \ldots, \bar{x}_{n}\right)$ with $\bar{x}_{i}=x_{i}^{-1}$ for $i=1,2, \ldots, n$ and let $\lambda=\left(\lambda_{1}, \lambda_{2}, \ldots, \lambda_{n}\right)$ be a partition of length $\ell(\lambda) \leq n$. Then each of the classical groups $G=G L(n, \mathbb{C}), S p(2 n, \mathbb{C})$ and $S O(2 n+1, \mathbb{C})$ possesses a finite dimensional irreducible representation $V_{G}^{\lambda}$ of highest weight $\lambda$ whose character may be denoted by $\operatorname{ch} V_{G}^{\lambda}(\mathbf{z})$ where $\mathbf{z}$ is a suitable parametrisation of the eigenvalues of the group elements of $G$, namely $\mathbf{x},(\mathbf{x}, \overline{\mathbf{x}})$ and $(\mathbf{x}, \overline{\mathbf{x}}, 1)$, respectively. We leave aside the somewhat more complicated case of $S O(2 n, \mathbb{C})$. The character $\operatorname{ch} V_{G L(n, \mathbb{C})}^{\lambda}(\mathbf{x})$ is none other than the Schur function $s_{\lambda}(\mathbf{x})$, and we adopt a similar notation for all our characters as follows:

$$
\operatorname{ch} V_{G L(n, \mathbb{C})}^{\lambda}(\mathbf{x})=s_{\lambda}(\mathbf{x}) ; \operatorname{ch} V_{S p(2 n, \mathbb{C})}^{\lambda}(\mathbf{x}, \overline{\mathbf{x}})=s p_{\lambda}(\mathbf{x}, \overline{\mathbf{x}}) ; \operatorname{ch} V_{S O(2 n+1, \mathbb{C})}^{\lambda}(\mathbf{x}, \overline{\mathbf{x}}, 1)=s o_{\lambda}(\mathbf{x}, \overline{\mathbf{x}}, 1) \text {. }
$$

Setting $\mathbf{a}=\left(a_{1}, a_{2}, \ldots\right)$, the transition from ordinary to factorial characters may be made through the judicious use of the map

$$
x^{m} \rightarrow \begin{cases}(x \mid \mathbf{a})^{m}=\left(x+a_{1}\right)\left(x+a_{2}\right) \cdots\left(x+a_{m}\right) & \text { if } m>0 ; \\ 1 & \text { if } m=0 ; \\ (\bar{x} \mid \mathbf{a})^{|m|}=\left(\bar{x}+a_{1}\right)\left(\bar{x}+a_{2}\right) \cdots\left(\bar{x}+a_{|m|}\right) & \text { if } m<0 .\end{cases}
$$


To be precise we propose the following definition of factorial characters of the classical Lie groups:

Definition 2 For $\mathbf{x}=\left(x_{1}, x_{2}, \ldots, x_{n}\right), \overline{\mathbf{x}}=\left(\bar{x}_{1}, \bar{x}_{2}, \ldots, \bar{x}_{n}\right)$ and $\mathbf{a}=\left(a_{1}, a_{2}, \ldots\right)$, let

$$
\begin{aligned}
s_{\lambda}(\mathbf{x} \mid \mathbf{a}) & =\frac{\left|\left(x_{i} \mid \mathbf{a}\right)^{\lambda_{j}+n-j}\right|}{\left|\left(x_{i} \mid \mathbf{a}\right)^{n-j}\right|} ; \\
s p_{\lambda}(\mathbf{x}, \overline{\mathbf{x}} \mid \mathbf{a}) & =\frac{\left|x_{i}\left(x_{i} \mid \mathbf{a}\right)^{\lambda_{j}+n-j}-\bar{x}_{i}\left(\bar{x}_{i} \mid \mathbf{a}\right)^{\lambda_{j}+n-j}\right|}{\left|x_{i}\left(x_{i} \mid \mathbf{a}\right)^{n-j}-\bar{x}_{i}\left(\bar{x}_{i} \mid \mathbf{a}\right)^{n-j}\right|} ; \\
s o_{\lambda}(\mathbf{x}, \overline{\mathbf{x}}, 1 \mid \mathbf{a}) & =\frac{\left|x_{i}^{1 / 2}\left(x_{i} \mid \mathbf{a}\right)^{\lambda_{j}+n-j}-\bar{x}_{i}^{1 / 2}\left(\bar{x}_{i} \mid \mathbf{a}\right)^{\lambda_{j}+n-j}\right|}{\left|x_{i}^{1 / 2}\left(x_{i} \mid \mathbf{a}\right)^{n-j}-\bar{x}_{i}^{1 / 2}\left(\bar{x}_{i} \mid \mathbf{a}\right)^{n-j}\right|} .
\end{aligned}
$$

The well-known formulae for the ordinary characters of the classical Lie groups may be recovered by setting $\mathbf{a}=(0,0, \ldots)$ which has the effect of reversing the map (2) and thereby reducing $\left(x_{i} \mid \mathbf{a}\right)^{m}$ and $\left(\bar{x}_{i} \mid \mathbf{a}\right)^{m}$ to $x_{i}^{m}$ and $x_{i}^{-m}$, respectively, for all $m \geq 0$.

\section{Flagged Jacob-Trudi identities}

To establish factorial Jacobi-Trudi identites we need analogues of the complete homogeneous symmetric functions $h_{r}(\mathbf{x})$ that are appropriate not only to the case of the other group characters but also to the case of our factorial characters. Just as is done classically for $h_{r}(\mathbf{x})$, it is convenient to define these analogues by means of generating functions. Each generating function $F_{m}(\mathbf{z} ; t)$ may be expanded as a power series in $t$, and we denote the coefficient of $t^{m}$ in such an expansion by $\left[t^{m}\right] F_{m}(\mathbf{z} ; t)$ for all integers $m$.

Definition 3 Let $\mathbf{x}=\left(x_{1}, x_{2}, \ldots, x_{n}\right), \overline{\mathbf{x}}=\left(\bar{x}_{1}, \bar{x}_{2}, \ldots, \bar{x}_{n}\right)$ and $\mathbf{a}=\left(a_{1}, a_{2}, \ldots\right)$. Then for any integer $m$ let

$$
\begin{aligned}
h_{m}(\mathbf{x} \mid \mathbf{a}) & =\left[t^{m}\right] \prod_{i=1}^{n} \frac{1}{1-t x_{i}} \prod_{j=1}^{n+m-1}\left(1+t a_{j}\right) ; \\
h_{m}^{s p}(\mathbf{x}, \overline{\mathbf{x}} \mid \mathbf{a}) & =\left[t^{m}\right] \prod_{i=1}^{n} \frac{1}{\left(1-t x_{i}\right)\left(1-t \bar{x}_{i}\right)} \prod_{j=1}^{n+m-1}\left(1+t a_{j}\right) ; \\
h_{m}^{s o}(\mathbf{x}, \overline{\mathbf{x}}, 1 \mid \mathbf{a}) & =\left[t^{m}\right](1+t) \prod_{i=1}^{n} \frac{1}{\left(1-t x_{i}\right)\left(1-t \bar{x}_{i}\right)} \prod_{j=1}^{n+m-1}\left(1+t a_{j}\right) .
\end{aligned}
$$

For $m=0$ we have $h_{0}(\mathbf{x} \mid \mathbf{a})=h_{0}^{s o}(\mathbf{x}, \overline{\mathbf{x}}, 1 \mid \mathbf{a})=h_{0}^{s p}(\mathbf{x}, \overline{\mathbf{x}} \mid \mathbf{a})=h_{0}^{o}(\mathbf{x}, \overline{\mathbf{x}} \mid \mathbf{a})=1$, while for $m<0$ we have $h_{m}(\mathbf{x} \mid \mathbf{a})=h_{m}^{s o}(\mathbf{x}, \overline{\mathbf{x}}, 1 \mid \mathbf{a})=h_{m}^{s p}(\mathbf{x}, \overline{\mathbf{x}} \mid \mathbf{a})=0$.

The one variable case $\mathbf{x}=\left(x_{i}\right)$ of Definition 3 allows us to rewrite our factorial characters as:

\section{Lemma 4}

$$
\begin{aligned}
s_{\lambda}(\mathbf{x} \mid \mathbf{a}) & =\left|h_{\lambda_{j}+n-j}\left(x_{i} \mid \mathbf{a}\right)\right| /\left|h_{n-j}\left(x_{i} \mid \mathbf{a}\right)\right| ; \\
s p_{\lambda}(\mathbf{x}, \overline{\mathbf{x}} \mid \mathbf{a}) & =\left|h_{\lambda_{j}+n-j}^{s p}\left(x_{i}, \bar{x}_{i} \mid \mathbf{a}\right)\right| /\left|h_{n-j}^{s p}\left(x_{i}, \bar{x}_{i} \mid \mathbf{a}\right)\right| ; \\
s o_{\lambda}(\mathbf{x}, \overline{\mathbf{x}}, 1 \mid \mathbf{a}) & =\left|h_{\lambda_{j}+n-j}^{s o}\left(x_{i}, \bar{x}_{i}, 1 \mid \mathbf{a}\right)\right| /\left|h_{n-j}^{s o}\left(x_{i}, \bar{x}_{i}, 1 \mid \mathbf{a}\right)\right| .
\end{aligned}
$$


Proof: In the case of $s_{\lambda}(\mathbf{x} \mid \mathbf{a})$ it suffices to note that for $m \geq 0$

$$
\begin{aligned}
& h_{m}\left(x_{i} \mid \mathbf{a}\right)=\left[t^{m}\right] \frac{1}{1-t x_{i}} \prod_{j=1}^{m}\left(1+t a_{j}\right)=\left[t^{m}\right] \frac{1+t a_{m}}{1-t x_{i}} \prod_{j=1}^{m-1}\left(1+t a_{j}\right) \\
& =\left[t^{m}\right]\left(1+\frac{t\left(x_{i}+a_{m}\right)}{1-t x_{i}}\right) \prod_{j=1}^{m-1}\left(1+t a_{j}\right)=\left(x_{i}+a_{m}\right)\left[t^{m-1}\right] \frac{1}{1-t x_{i}} \prod_{j=1}^{m-1}\left(1+t a_{j}\right) \\
& =\left(x_{i}+a_{m}\right)\left(x_{i}+a_{m-1}\right) \cdots\left(x_{i}+a_{1}\right)\left[t^{0}\right] \frac{1}{1-t x_{i}}=\left(x_{i} \mid \mathbf{a}\right)^{m} .
\end{aligned}
$$

One then just uses this identity in 3 with $m=\lambda_{j}+n-j$ and $m=n-j$ in the numerator and denominator, respectively. Then in the case of $s p_{\lambda}(\mathbf{x}, \overline{\mathbf{x}} \mid \mathbf{a})$ we have

$$
\begin{aligned}
& h_{m}^{s p}\left(x_{i}, \bar{x}_{i} \mid \mathbf{a}\right)=\left[t^{m}\right] \frac{1}{\left(1-t x_{i}\right)\left(1-t \bar{x}_{i}\right)} \prod_{j=1}^{m}\left(1+t a_{j}\right) \\
& =\left[t^{m}\right] \frac{1}{x_{i}-\bar{x}_{i}}\left(\frac{x_{i}}{1-t x_{i}}-\frac{\bar{x}_{i}}{1-t \bar{x}_{i}}\right) \prod_{j=1}^{m}\left(1+t a_{j}\right)=\frac{1}{x_{i}-\bar{x}_{i}}\left(x_{i}\left(x_{i} \mid \mathbf{a}\right)^{m}-\bar{x}_{i}\left(\bar{x}_{i} \mid \mathbf{a}\right)^{m}\right),
\end{aligned}
$$

using (12). Applying this to the numerator and denominator of (4) with $m=\lambda_{j}+n-j$ and $m=n-j$, the result follows from cancellation of factors $x_{i}-\bar{x}_{i}$. The orthogonal case is similar.

Now we may transform each expression in Lemma 4 into flagged Jacobi-Trudi identities via:

Lemma 5 For $1 \leq i<j \leq n$ and all integers $m$ :

$$
\begin{array}{r}
h_{m}\left(x_{i}, \ldots x_{j-1} \mid \mathbf{a}\right)-h_{m}\left(x_{i+1}, \ldots, x_{j} \mid \mathbf{a}\right) \\
\quad=\left(x_{i}-x_{j}\right) h_{m-1}\left(x_{i}, \ldots, x_{j} \mid \mathbf{a}\right) \\
h_{m}^{s p}\left(x_{i}, \bar{x}_{i}, \ldots, x_{j-1}, \bar{x}_{j-1} \mid \mathbf{a}\right)-h_{m}^{s p}\left(x_{i+1}, \bar{x}_{i+1}, \ldots, x_{j}, \bar{x}_{j}, \mid \mathbf{a}\right) \\
\quad=\left(x_{i}-x_{j}\right)\left(1-\bar{x}_{i} \bar{x}_{j}\right) h_{m-1}^{s p}\left(x_{i}, \bar{x}_{i}, \ldots, x_{j}, \bar{x}_{j}, \mid \mathbf{a}\right) \\
h_{m}^{s o}\left(x_{i}, \bar{x}_{i}, \ldots, x_{j-1}, \bar{x}_{j-1}, 1 \mid \mathbf{a}\right)-h_{m}^{s o}\left(x_{i+1}, \bar{x}_{i+1}, \ldots, x_{j}, \bar{x}_{j}, 1 \mid \mathbf{a}\right) \\
=\left(x_{i}-x_{j}\right)\left(1-\bar{x}_{i} \bar{x}_{j}\right) h_{m-1}^{s o}\left(x_{i}, \bar{x}_{i}, \ldots, x_{j}, \bar{x}_{j}, 1 \mid \mathbf{a}\right) .
\end{array}
$$

Proof: First note these identities are trivially true for $m<0$ and $m=0$ since each $h_{m}$ reduces to either 0 or 1 , and each $h_{m-1}$ to 0 . For $m>0$ all cases are similar and we illustrate just the symplectic case:

$$
\begin{aligned}
& h_{m}^{s p}\left(x_{i}, \bar{x}_{i}, \ldots, x_{j-1}, \bar{x}_{j-1} \mid \mathbf{a}\right)-h_{m}^{s p}\left(x_{i+1}, \bar{x}_{i+1}, \ldots, x_{j}, \bar{x}_{j}, \mid \mathbf{a}\right) \\
& =\left[t^{m}\right]\left(\left(1-t x_{j}\right)\left(1-t \bar{x}_{j}\right)-\left(1-t x_{i}\right)\left(1-t \bar{x}_{i}\right)\right) \prod_{\ell=i}^{j} \frac{1}{\left(1-t x_{\ell}\right)\left(1-t \bar{x}_{\ell}\right)} \prod_{k=1}^{m+j-i-1}\left(1+t a_{k}\right) \\
& =\left(x_{i}+\bar{x}_{i}-x_{j}-\bar{x}_{j}\right)\left[t^{m-1}\right] \prod_{\ell=i}^{j} \frac{1}{\left(1-t x_{\ell}\right)\left(1-t \bar{x}_{\ell}\right)} \prod_{k=1}^{(m-1)+j-i}\left(1+t a_{k}\right) \\
& =\left(x_{i}-x_{j}\right)\left(1-\bar{x}_{i} \bar{x}_{j}\right) h_{m-1}^{s p}\left(x_{i}, \bar{x}_{i}, \ldots, x_{j}, \bar{x}_{j}, \mid \mathbf{a}\right) .
\end{aligned}
$$


Now we are a position to state and prove the following result (see Okada [14] and Chen et al. [5] for the non-factorial case).

Theorem 6 (Flagged Jacobi-Trudi identity) Given $\mathbf{x}=\left(x_{1}, x_{2}, \ldots, x_{n}\right)$ and $\overline{\mathbf{x}}=\left(\bar{x}_{1}, \bar{x}_{2}, \ldots, \bar{x}_{n}\right)$ with $\bar{x}_{k}=x_{k}^{-1}$ for $k=1,2, \ldots, n$, let $\mathbf{x}^{(i)}=\left(x_{i}, x_{i+1}, \ldots, x_{n}\right)$ and $\overline{\mathbf{x}}^{(i)}=\left(\bar{x}_{i}, \bar{x}_{i+1}, \ldots, \bar{x}_{n}\right)$. Then for any partition $\lambda=\left(\lambda_{1}, \lambda_{2}, \ldots, \lambda_{n}\right)$ and any $\mathbf{a}=\left(a_{1}, a_{2}, \ldots\right)$ including the case $\mathbf{a}=(0,0, \ldots)$ we have

$$
\begin{aligned}
s_{\lambda}(\mathbf{x} \mid \mathbf{a}) & =\left|h_{\lambda_{j}-j+i}\left(\mathbf{x}^{(i)} \mid \mathbf{a}\right)\right| \\
s p_{\lambda}(\mathbf{x}, \overline{\mathbf{x}} \mid \mathbf{a}) & =\left|h_{\lambda_{j}-j+i}^{s p}\left(\mathbf{x}^{(i)}, \overline{\mathbf{x}}^{(i)} \mid \mathbf{a}\right)\right| ; \\
s o_{\lambda}(\mathbf{x}, \overline{\mathbf{x}}, 1 \mid \mathbf{a}) & =\left|h_{\lambda_{j}-j+i}^{s o}\left(\mathbf{x}^{(i)}, \overline{\mathbf{x}}^{(i)}, 1 \mid \mathbf{a}\right)\right| .
\end{aligned}
$$

Proof: Focussing on the symplectic case, we proceed by manipulating the determinant in the numerator of 10 , subtracting row $(i+1)$ from row $i$ for $i=1,2, \ldots, n-1$ and applying $[15]$, then repeating the process for $i=1,2, \ldots, n-2$, and so on. This yields

$$
\left|h_{\lambda_{j}+n-j}^{s p}\left(x_{i} \mid \mathbf{a}\right)\right|=\prod_{1 \leq i<j \leq n}\left(x_{i}-x_{j}\right)\left(1-\bar{x}_{i} \bar{x}_{j}\right)\left|h_{\lambda_{j}-j+i}^{s p}\left(\mathbf{x}^{(i)}, \overline{\mathbf{x}}^{(i)} \mid \mathbf{a}\right)\right| .
$$

Dividing this by the same expression with $\lambda=(0)$ gives $(19)$ since in this case the right-hand determinant of 21 is lower triangular with diagonal elements all 1 . The other two results follow in the same way.

\section{Explicit formulae in the case of one part partitions}

As a consequence of Theorem 6 it should be noted that we have

Corollary 7 In the special case $\lambda=(m, 0, \ldots, 0)$

$$
s_{(m)}(\mathbf{x} \mid \mathbf{a})=h_{m}(\mathbf{x} \mid \mathbf{a}) ; \quad s p_{(m)}(\mathbf{x}, \overline{\mathbf{x}} \mid \mathbf{a})=h_{m}^{s p}(\mathbf{x}, \overline{\mathbf{x}} \mid \mathbf{a}) ; \quad s O_{(m)}(\mathbf{x}, \overline{\mathbf{x}}, 1 \mid \mathbf{a})=h_{m}^{s o}(\mathbf{x}, \overline{\mathbf{x}}, 1 \mid \mathbf{a}) .
$$

Proof: On setting $\lambda=(m, 0, \ldots, 0)$ the flagged Jacobi-Trudi determinants in Theorem 6 are reduced to lower-triangular form since each $h_{-j+i}=0$ for $i<j$. Moreover for $i>1$ the diagonal entries are all of the form $h_{0}=1$, while the $(1,1)$ entry is just $h_{m}$ with $\mathbf{x}^{(1)}=\mathbf{x}$ and $\overline{\mathbf{x}}^{(1)}=\overline{\mathbf{x}}$.

Factorial characters in the one-part partition case may then be evaluated directly from the generating function formulae of Definition 3 In the Schur function case with $\mathbf{x}=\left(x_{1}, x_{2}, \ldots, x_{n}\right)$ and $\mathbf{x}^{\prime}=$ $\left(x_{1}, x_{2}, \ldots, x_{n-1}\right)$ one finds $h_{m}(\mathbf{x} \mid \mathbf{a})=h_{m}\left(\mathbf{x}^{\prime} \mid \mathbf{a}\right)+\left(x_{n}+a_{m+n-1}\right) h_{m-1}(\mathbf{x} \mid \mathbf{a})$. Iterating this recurrence relation gives

$$
h_{m}(\mathbf{x} \mid \mathbf{a})=\sum_{1 \leq i_{1} \leq i_{2} \leq \cdots \leq i_{m} \leq n}\left(x_{i_{1}}+a_{i_{1}}\right)\left(x_{i_{2}}+a_{i_{2}+1}\right) \cdots\left(x_{i_{m}}+a_{i_{m}+m-1}\right) .
$$


This result can be exploited in the symplectic case, where it might be noted first that if we introduce dummy parameters $a_{\ell}=0$ for $\ell=0,-1,-2, \ldots$ we have

$$
h_{m}^{s p}(\mathbf{x}, \overline{\mathbf{x}} \mid \mathbf{a})=\left[t^{m}\right] \prod_{i=1}^{n} \frac{1}{\left(1-t x_{i}\right)\left(1-t \bar{x}_{i}\right)} \prod_{k=1-n}^{m+2 n-1-n}\left(1+t a_{k}\right)=h_{m}\left(\mathbf{z} \mid \tau^{-n} \mathbf{a}\right) .
$$

with $\mathbf{z}=\left(x_{1}, \bar{x}_{1}, x_{2}, \bar{x}_{2}, \ldots, x_{n}, \bar{x}_{n}\right)$ and $\tau^{-n} \mathbf{a}=\left(a_{-n+1}, \ldots, a_{-1}, a_{0}, a_{1}, a_{2} \ldots\right)$. It follows that

$$
h_{m}^{s p}(\mathbf{x}, \overline{\mathbf{x}} \mid \mathbf{a})=\sum_{1 \leq i_{1} \leq i_{2} \leq \cdots \leq i_{m} \leq 2 n}\left(z_{i_{1}}+a_{i_{1}-n}\right)\left(z_{i_{2}}+a_{i_{2}-n+1}\right) \cdots\left(z_{i_{m}}+a_{i_{m}-n+m-1}\right) .
$$

where $z_{i_{j}}+a_{i_{j}-n+j-1}=\left\{\begin{array}{ll}x_{k}+a_{2 k-n+j-2} & \text { if } i_{j}=2 k-1 ; \\ \bar{x}_{k}+a_{2 k-n+j-1} & \text { if } i_{j}=2 k,\end{array}\right.$ with $a_{\ell}=0$ if $\ell \leq 0$. The odd orthogonal case is similar but with the inclusion of a factor $\left(1-a_{n+j}\right)$ if $i_{m}=2 n+1$.

\section{Combinatorial realisation of factorial characters}

The significance of these results is that they offer an immediate lattice path model of each of the relevant one-part partition factorial characters. By making use of $n$-tuples of such lattice paths in the interpretation of the flagged Jacobi-Trudi identities of Theorem 6 one arrives at a non-intersecting lattice path model of factorial characters specified by any partition $\lambda$ of length $\ell(\lambda) \leq n$. This leads inexorably to a further realisation of factorial characters in terms of certain appropriately weighted tableaux. The tableaux themselves are none other than those already associated with Schur functions, symplectic group characters and odd orthogonal group characters in the classical non-factorial case.

Restricting our attention to fixed $n$ and partitions $\lambda=\left(\lambda_{1}, \lambda_{2}, \ldots, \lambda_{n}\right)$ of length $\ell(\lambda) \leq n$, each such partition defines a Young diagram $F^{\lambda}$ consisting of $|\lambda|=\lambda_{1}+\lambda_{2}+\cdots+\lambda_{n}$ boxes arranged in $\ell(\lambda)$ rows of lengths $\lambda_{i}$. For $i=1,2, \ldots, \ell(\lambda)$. Let $(i, j)$ signify the box in the $i$ th row and $j$ th column of $F^{\lambda}$.

Definition 8 Let $\mathcal{T}_{\lambda}$ be the set of all semistandard Young tableaux $T$ of shape $\lambda$ that are obtained by filling each box $(i, j)$ of $F^{\lambda}$ with an entry $T_{i j}$ from the alphabet $\{1<2<\cdots<n\}$ in all possible ways such that: (T1) entries weakly increase across rows from left to right; (T2) entries strictly increase down columns from top to bottom.

Definition 9 [11] Let $\mathcal{T}_{\lambda}^{\text {sp }}$ be the set of all symplectic tableau $T$ of shape $\lambda$ that are obtained by filling each box $(i, j)$ of $F^{\lambda}$ with an entry $T_{i j}$ from the alphabet $\{1<\overline{1}<2<\overline{2}<\cdots<n<\bar{n}\}$ in all possible ways such that: (Sp1) entries weakly increase across each row from left to right; (Sp2) entries strictly increase down each column from top to bottom; (Sp3) neither $k$ nor $\bar{k}$ appear lower than the kth row.

Definition $10[18]$ [ Let $\mathcal{T}_{\lambda}^{\text {so }}$ be the set of all odd orthogonal tableaux $T$ of shape $\lambda$ obtained by filling each box $(i, j)$ of $F^{\lambda}$ with an entry $T_{i j}$ from the alphabet $\{1<\overline{1}<2<\overline{2}<\cdots<n<\bar{n}<0\}$ in all possible ways such that: (O1) entries weakly increase across each row from left to right; (O2) entries weakly increase down each column from top to bottom; (O3) neither $k$ nor $\bar{k}$ appear lower than the $k$ th row; (04) no two non-zero entries in any column are equal; (05) in any row 0 appears at most once.

These definitions allow combinatorial expressions of our factorial characters as follows: 
Theorem 11 For each $g$ and $\mathbf{z}$ as tabulated below,

$$
g_{\lambda}(\mathbf{z} \mid \mathbf{a})=\sum_{T \in \mathcal{T}_{\lambda}^{g}} \prod_{(i, j) \in F^{\lambda}} \operatorname{wgt}\left(T_{i j}\right), \text { where }
$$

\begin{tabular}{|l|l|l|l|}
\hline$g_{\lambda}(\mathbf{z} \mid \mathbf{a})$ & $T_{i j}$ & $\operatorname{wgt}\left(T_{i j}\right)$ & \\
\hline$s_{\lambda}(\mathbf{x} \mid \mathbf{a})$ & $k$ & $x_{k}+a_{k+j-i}$ & \\
\hline$s p_{\lambda}(\mathbf{x}, \overline{\mathbf{x}} \mid \mathbf{a})$ & $\frac{k}{k}$ & $x_{k}+a_{2 k-1-n+j-i}$ & $a_{m}=0$ for $m \leq 0$ \\
& $\bar{x}_{k}+a_{2 k-n+j-i}$ & \\
\hline$s o_{\lambda}(\mathbf{x}, \overline{\mathbf{x}}, 1 \mid \mathbf{a})$ & $k$ & $x_{k}+a_{2 k-n+j-i}$ & $a_{m}=0$ for $m \leq 0$ \\
& $\bar{k}$ & $\bar{x}_{k}+a_{2 k+1-n+j-i}$ & \\
& 0 & $1-a_{n+1+j-i}$ & \\
\hline
\end{tabular}

Proof: In the Schur function case, as in [8], we adopt matrix coordinates $(k, \ell)$ for lattice points with $k=1,2, \ldots, n$ specifying row labels from top to bottom, and $\ell=1,2, \ldots, \lambda_{1}+n$ specifying column labels from left to right. Each lattice path that we are interested in is a continuous path from some $P_{i}=(i, n-i+1)$ to some $Q_{j}=\left(n, n-j+1+\lambda_{j}\right)$. Such a path consists of a sequence of horizontal or vertical edges and is associated with a contribution to $h_{\lambda_{j}-j+i}\left(\mathbf{x}^{(i)} \mid \mathbf{a}\right)$ in the form of a summand of 23 with $m=\lambda_{j}-j+i$ and $\mathbf{x}$ replaced by $\mathbf{x}^{(i)}$. Taking into account the restriction of alphabet from $\mathbf{x}$ to $\mathbf{x}^{(i)}$, the weight assigned to a horizontal edge from $(k, \ell-1)$ to $(k, \ell)$ is $x_{k}+a_{k+\ell-n-1}$. Thanks to the Lindström-Gessel-Viennot theorem the only surviving contributions to the determinantal expression for $s_{\lambda}(\mathbf{x} \mid \mathbf{a})$ in the flagged Jacobi-Trudi identity $(18)$ are those correspnding to an $n$-tuple of non-intersecting lattice paths from $P_{i}$ to $Q_{i}$ for $i=1,2, \ldots, n$. Such $n$-tuples are easily seen to be in bijective correspondence with semistandard Young tableaux $T$ of shape $\lambda$ as in Definition 8 , with the $j$ th horizontal edge at level $k$ on the path from $P_{i}$ to $Q_{i}$ giving an entry $T_{i j}=k$ in $T$ for $i=1,2, \ldots, n$ and $j=1,2, \ldots, \lambda_{i}$. To complete the proof of Theorem 11 in the factorial Schur function case it only remains to note that the weight $\operatorname{wgt}\left(T_{i j}\right)$ to be assigned to $T_{i j}$ is that of the edge from $(k, \ell-1)$ to $(k, \ell)$ given by $x_{k}+a_{k+\ell-n-1}=x_{k}+a_{k+j-i}$ with $j=\ell-(n-i+1)$ since this is the number of horizontal steps from $P_{i}$ to column $\ell$ on the lattice path from $P_{i}$ to $Q_{i}$. This is exemplified in Fig. 1 of [8].

In the factorial symplectic case the lattice path proof proceeds exactly as in the Schur function case with the alphabet extended to include both $x_{k}$ and $\bar{x}_{k}$, with a replaced by $\tau^{-n}$ a as dictated by (24) and with the starting points $P_{i}=(i, n-i+1)$ replaced by $P_{i}=(2 i-1, n-i+1)$ to ensure that condition $(\mathbf{S p 3})$ is satisfied. Once again it is only the $n$-tuples of non-intersecting lattice paths from $P_{i}$ to $Q_{i}$ that contribute to $s p_{\lambda}(\mathbf{x}, \overline{\mathbf{x}} \mid \mathbf{a})$ and these are in bijective correspondence with the symplectic tableaux $T$ of shape $\lambda$ with entries from $\{1<\overline{1}<\cdots<n<\bar{n}\}$. This is exemplified in Figure 1 for $n=4$ and $\lambda=(4,3,3)$.

The argument in the odd orthogonal factorial case proceeds very much as before but this time diagonal rather than horizontal edges appear at level $2 k+1$.

\section{Tokuyama type identities}

A partition is said to be strict if its non-zero parts are distinct. Each such strict partition $\lambda$ of length $\ell(\lambda) \leq$ $n$ specifies a shifted Young diagram $S F^{\lambda}$ consisting of rows of boxes of lengths $\lambda_{i}$ for $i=1,2, \ldots, \ell(\lambda)$ left adjusted to a diagonal line. This allows us to define various primed shifted tableaux. 


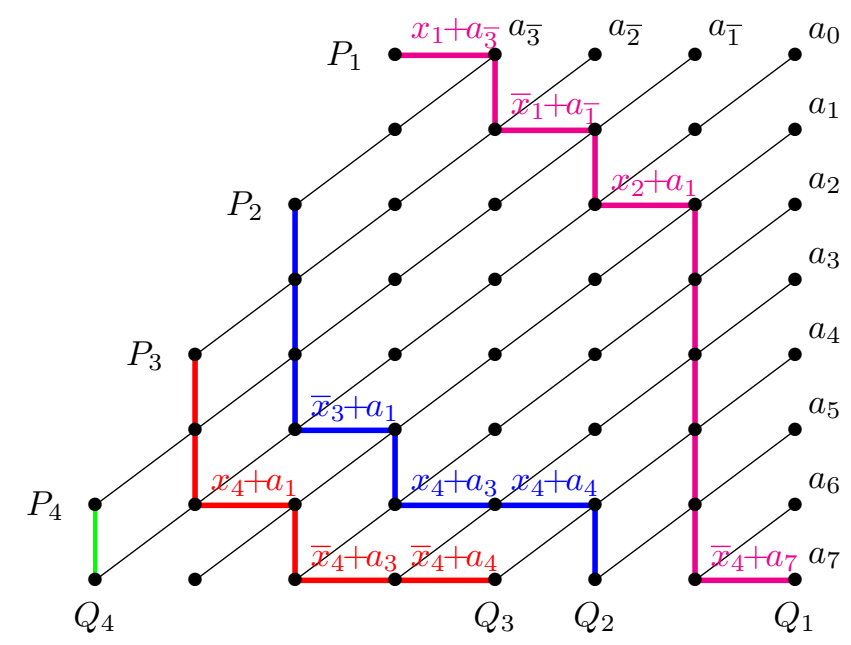

\begin{tabular}{|c|c|c|c|}
\hline$x_{1}$ & $\bar{x}_{1}$ & $x_{2}+a_{1}$ & $x_{\overline{4}}+a_{7}$ \\
\hline$x_{\overline{3}}+a_{1}$ & $x_{4}+a_{3}$ & $x_{4}+a_{4}$ & \\
\cline { 1 - 2 }$x_{4}+a_{1}$ & $x_{\overline{4}}+a_{3}$ & $x_{\overline{4}}+a_{4}$ & \\
\end{tabular}

Fig. 1: Example of non-intersecting lattice paths and a corresponding symplectic tableau and its factorial weights.

Definition 12 [16 20] Let $\mathcal{P}_{\lambda}^{g l}$ be the set of all primed shifted tableaux $P$ of shape $\lambda$ that are obtained by filling each box of $S F^{\lambda}$ with an entry $P_{i j}$ from the alphabet $\left\{1^{\prime}<1<2^{\prime}<2<\cdots<n^{\prime}<n\right\}$ with one entry in each box, in such a way that: (Q1) entries weakly increase from left to right across rows; (Q2) entries weakly increase from top to bottom down columns; (Q3) no two identical unprimed entries appear in any column; (Q4) no two identical primed entries appear in any row;

Definition 13 [7] Let $\mathcal{P}_{\lambda}^{s p}$ be the set of all primed shifted tableaux $P$ of shape $\lambda$ that are obtained by filling each box of $S F^{\lambda}$ with an entry $P_{i j}$ from the alphabet $\left\{1^{\prime}<1<\overline{1}^{\prime}<\overline{1}<2^{\prime}<2<\overline{2}^{\prime}<\overline{2}<\cdots<\right.$ $\left.n^{\prime}<n<\bar{n}^{\prime}<\bar{n}\right\}$ with one entry in each box, in such a way that the conditions (Q1)-(Q4) are satisfied together with: (Q5) at most one of $\left\{k^{\prime}, k, \bar{k}^{\prime}, \bar{k}\right\}$ appears on the main diagonal for each $k=1,2, \ldots, n$.

Definition 14 Let $\mathcal{P}_{\lambda}^{\text {so }}$ be the set of all primed shifted tableaux $P$ of shape $\lambda$ that are obtained by filling each box of $S F^{\lambda}$ with an entry $P_{i j}$ from the alphabet $\left\{1^{\prime}<1<\overline{1}^{\prime}<\overline{1}<2^{\prime}<2<\overline{2}^{\prime}<\overline{2}<\cdots<n^{\prime}<\right.$ $\left.n<\bar{n}^{\prime}<\bar{n}<0^{\prime}\right\}$ with one entry in each box, in such a way that the conditions (Q1)-(Q5) are satisfied. together with: (Q6) the entry $0^{\prime}$ does not appear on the main diagonal.

Our proposed definition of factorial $Q$-functions then takes the form

Definition 15 For $\mathbf{a}=\left(a_{1}, a_{2}, \ldots\right), a_{0}=0$ and any strict partition $\lambda$ of length $\ell(\lambda) \leq n$, let

$$
Q_{\lambda}^{g}(\mathbf{z} ; \mathbf{w} \mid \mathbf{a})=\sum_{P \in \mathcal{P}_{\lambda}^{g}} \prod_{(i, j) \in S F^{\lambda}} \operatorname{wgt}\left(P_{i j}\right) \quad \text { where }
$$

\begin{tabular}{|l|l|}
\hline$g$ & $Q_{\lambda}^{g}(\mathbf{z} ; \mathbf{w} \mid \mathbf{a})$ \\
\hline$g l$ & $Q_{\lambda}(\mathbf{x} ; \mathbf{y} \mid \mathbf{a})$ \\
$s p$ & $Q_{\lambda}^{s p}(\mathbf{x}, \overline{\mathbf{x}} ; \mathbf{y}, \overline{\mathbf{y}} \mid \mathbf{a})$ \\
$s o$ & $Q_{\lambda}^{s o}(\mathbf{x}, \overline{\mathbf{x}} ; \mathbf{y}, \overline{\mathbf{y}}, 1 \mid \mathbf{a})$ \\
\hline
\end{tabular}

and

\begin{tabular}{|l|l||l|l|}
\hline$P_{i j}$ & $\operatorname{wgt}\left(P_{i j}\right)$ & $P_{i j}$ & $\operatorname{wgt}\left(P_{i j}\right)$ \\
\hline$k$ & $x_{k}+a_{j-i}$ & $k^{\prime}$ & $y_{k}-a_{j-i}$ \\
$\bar{k}$ & $\bar{x}_{k}+a_{j-i}$ & $\bar{k}^{\prime}$ & $\bar{y}_{k}-a_{j-i}$ \\
& & $0^{\prime}$ & $1-a_{j-i}$ \\
\hline
\end{tabular}


This definition includes the case $Q_{\lambda}(\mathbf{x} ; \mathbf{y} \mid a)$ introduced previously [8] and that is here the subject of Theorem 1. To prove factorial versions of Tokuyama's identity for these $Q$-functions for $\ell(\lambda)=n$ we need an intermediate result that exploits lattice paths to express each $Q$-function as a single determinant.

\begin{tabular}{|c|c|c|c|c|c|c|c|}
\hline$(i, i)$ & $i$ & $x_{i}$ & $\ddots$ & $(i, i)$ & $i^{\prime}$ & $y_{i}$ & $\bullet$ \\
\hline$(i, i)$ & $\bar{i}$ & $\bar{x}_{i}$ & $\bullet$ & $(i, i)$ & $\bar{i}^{\prime}$ & $\bar{y}_{i}$ & \\
\hline$(i, j) i<j$ & $k$ & $x_{k}+a_{j-i}$ & $\bullet$ & $(i, j) i<j$ & $k^{\prime}$ & $y_{k}-a_{j-i}$ & \\
\hline$(i, j) i<j$ & $\bar{k}$ & $\bar{x}_{k}+a_{j-i}$ & $\bullet$ & $(i, j) i<j$ & $\bar{k}^{\prime}$ & $\bar{y}_{k}-a_{j-i}$ & \\
\hline
\end{tabular}

Fig. 2: Edges and their weights in the factorial symplectic $Q$-function case.

Lemma 16 For any strict partition $\lambda$ of length $\ell(\lambda)=n$,

$$
\begin{aligned}
Q_{\lambda}(\mathbf{x} ; \mathbf{y} \mid \mathbf{a}) & =\left|\left(x_{i}+y_{i}\right) q_{\lambda_{j}-1}^{g l}\left(\mathbf{x}^{(i)} ; \mathbf{y}^{(i+1)} \mid \mathbf{a}\right)\right| ; \\
Q_{\lambda}^{s p}(\mathbf{x}, \overline{\mathbf{x}} ; \mathbf{y}, \overline{\mathbf{y}} \mid \mathbf{a}) & =\left|\left(x_{i}+y_{i}+\bar{x}_{i}+\bar{y}_{i}\right) q_{\lambda_{j}-1}^{s p}\left(\mathbf{x}^{(i)}, \overline{\mathbf{x}}^{(i)} ; \mathbf{y}^{(i+1)}, \overline{\mathbf{y}}^{(i+1)} \mid \mathbf{a}\right)\right| ; \\
Q_{\lambda}^{s o}(\mathbf{x}, \overline{\mathbf{x}} ; \mathbf{y}, \overline{\mathbf{y}}, 1 \mid \mathbf{a})=\left|\left(x_{i}+y_{i}+\bar{x}_{i}+\bar{y}_{i}\right) q_{\lambda_{j}-1}^{s o}\left(\mathbf{x}^{(i)}, \overline{\mathbf{x}}^{(i)} ; \mathbf{y}^{(i+1)}, \overline{\mathbf{y}}^{(i+1)}, 1 \mid \mathbf{a}\right)\right|, & \\
\text { with } \quad q_{m}^{g}\left(\mathbf{x}^{(i)} ; \mathbf{y}^{(i+1)} \mid \mathbf{a}\right) & =\sum_{1 \leq i_{1} \leq i_{2} \leq \cdots \leq i_{m} \leq n} \sum_{\mathbf{z}}\left(z_{i_{1}} \pm a_{1}\right)\left(z_{i_{2}} \pm a_{2}\right) \cdots\left(z_{i_{m}} \pm a_{m}\right),
\end{aligned}
$$

where the sum over $\mathbf{z}$ allows factors $\left(z_{k} \pm a_{\ell}\right)=\left(x_{k}+a_{\ell}\right),\left(y_{k}-a_{\ell}\right)$ or $\left(1-a_{\ell}\right)$ to appear according as $z_{k}=x_{k}$, $y_{k}$, or 1 , with several factors of the form $\left(x_{k}+a_{\ell}\right)\left(x_{k}+a_{\ell+1}\right) \cdots$ allowed for any $k$ with $i \leq k \leq n$ but at most one factor $\left(y_{k}-a_{\ell}\right)$ for any $k$ with $i+1 \leq k \leq n$, and at most one factor $\left(1-a_{\ell}\right)$.

Proof: Each primed symplectic shifted tableau $P \in \mathcal{P}_{\lambda}^{s p}$ defines an $n$-tuple of non-intersecting lattice paths. The entries $P_{i j}$ in the $i$ th row of $P$ define a path from $P_{i}=\left(2 i-\frac{1}{2}, 0\right)$ to $Q_{i}=\left(2 n, \lambda_{i}\right)$. Since $\ell(\lambda)=n$ we have $P_{i i} \in\left\{i^{\prime}, i, \bar{i}^{\prime}, \bar{i}\right\}$ and these entries are curved edges from $P_{i}=\left(2 i-\frac{1}{2}, 0\right)$ to $(2 i-1,1)$ and $(2 i, 1)$ according as $P_{i i}$ is unbarred or barred, respectively, with the curve concave down or up according as $P_{i i}$ is unprimed or primed, respectively. For $j>i$ the $(j-i)$ th edge of the path $P_{i} Q_{i}$ is determined by the value of $P_{i j} \in\left\{k^{\prime}, k, \bar{k}^{\prime}, \bar{k}\right\}$ for some $k \geq i$ and is horizontal or diagonal according as $P_{i j}$ is unprimed or primed, respectively, and terminates at $(2 k-1, j-i)$ or $(2 k, j-i)$ according as $P_{i j}$ is unbarred or barred. See Fig. 2 for the edges and their weights (vertical edges have wgt 1). It is easy to see that the symplectic primed shifted tableaux $P \in \mathcal{P}_{\lambda}^{s p}$ are in bijective correspondence with $n$-tuples of non-intersecting lattice paths of the type described with start points $P_{i}$ and end points $Q_{i}$. See Fig. 3 . 


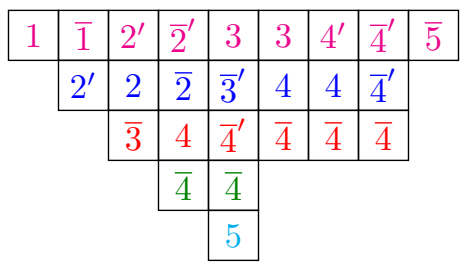

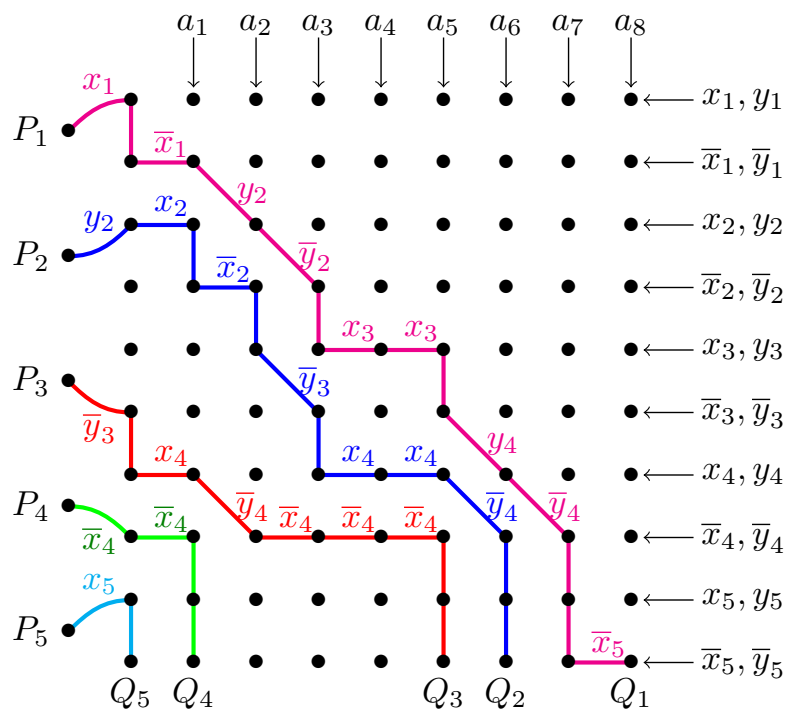

Fig. 3: A symplectic primed shifted tableau and the corresponding $n$-tuple of non-interesecting lattice paths.

A determinantal expression for $Q_{\lambda}^{s p}(\mathbf{x}, \overline{\mathbf{x}} ; \mathbf{y}, \overline{\mathbf{y}} \mid \mathbf{a})$ is obtained by considering $n$-tuples of paths from $P_{i}$ to $Q_{j}$ with $j=\pi(i)$ for all permutations $\pi \in S_{n}$. In this determinant the $(i, j)$ th element takes the form:

$$
\begin{aligned}
& \left(x_{i}+y_{i}\right) q_{\lambda_{j}-1}^{g l}\left(x^{(i)}, \bar{x}^{(i)} ; y^{(i+1)}, \bar{y}^{(i)} \mid \mathbf{a}\right)+\left(\bar{x}_{i}+\bar{y}_{i}\right) q_{\lambda_{j}-1}^{g l}\left(x^{(i+1)}, \bar{x}^{(i)} ; y^{(i+1)}, \bar{y}^{(i+1)} \mid \mathbf{a}\right) \\
& \quad=\left(x_{i}+y_{i}+\bar{x}_{i}+\bar{y}_{i}\right) q_{\lambda_{j}-1}^{s p}\left(x^{(i)}, \bar{x}^{(i)} ; y^{(i+1)}, \bar{y}^{(i+1)} \mid \mathbf{a}\right)
\end{aligned}
$$

Factors $\left(x_{i}+y_{i}\right)$ and $\left(\bar{x}_{i}+\bar{y}_{i}\right)$ arise since a path from $P_{i}$ starts with a curved edge of Fig. 2 The sum over all possible successive edges is then given by $q_{\lambda_{j}-1}^{g l}$ restricted to $\left\{i<\bar{i}^{\prime}<\bar{i}<(i+1)^{\prime}<\cdots, \bar{n}\right\}$ and $\left\{\bar{i}<(i+1)^{\prime},(i+1), \bar{i}^{\prime}<\cdots<\bar{n}\right\}$ (unprimed but not primed repetitions are allowed). The final identity is from the corresponding generating functions, but space does not allow its derivation.

At this point, for all $p$ and $q$ such that $1 \leq p \leq q \leq n$, it is convenient to introduce

$$
g_{m ; p, q, n}(\mathbf{x}, \overline{\mathbf{x}} ; \mathbf{y}, \overline{\mathbf{y}} \mid \mathbf{a})=\left[t^{m}\right] \frac{\prod_{j=q+1}^{n}\left(1+t y_{j}\right)\left(1+t \bar{y}_{j}\right)}{\prod_{i=p}^{n}\left(1-t x_{i}\right)\left(1-t \bar{x}_{i}\right)} \prod_{k=1}^{m+q-p}\left(1+t a_{k}\right) .
$$

This definition is such that:

$$
g_{m ; i, i, n}(\mathbf{x}, \overline{\mathbf{x}} ; \mathbf{y}, \overline{\mathbf{y}} \mid \mathbf{a})=q_{m}^{s p}\left(x^{(i)}, \bar{x}^{(i)} ; y^{(i+1)}, \bar{y}^{(i+1)} \mid \mathbf{a}\right) ;
$$




$$
\begin{aligned}
& g_{m ; i, n, n}(\mathbf{x}, \overline{\mathbf{x}} ; \mathbf{y}, \overline{\mathbf{y}} \mid \mathbf{a})=h_{m}^{s p}\left(x^{(i)}, \bar{x}^{(i)} \mid \mathbf{a}\right) \\
& g_{m, p, q-1, n}(x, \bar{x} ; y, \bar{y} \mid \mathbf{a})-g_{m, p+1, q, n}(x, \bar{x} ; y, \bar{y} \mid \mathbf{a}) \\
& \quad=\left(x_{p}+y_{q}+\bar{x}_{p}+\bar{y}_{q}\right) g_{m-1, p, q, n}(x, \bar{x} ; y, \bar{y} \mid \mathbf{a}) \text { if } p<q .
\end{aligned}
$$

Theorem 17 (Factorial Tokuyama Identities) Let $\lambda=\mu+\delta$ with $\delta=(n, n-1, \ldots, 1)$ and $\mu$ a partition of length $\ell(\mu) \leq n$. Then

$$
\begin{aligned}
Q_{\lambda}(\mathbf{x} ; \mathbf{y} \mid \mathbf{a}) & =\prod_{1 \leq i \leq j \leq n}\left(x_{i}+y_{j}\right) s_{\mu}(\mathbf{x} \mid \mathbf{a}) ; \\
Q_{\lambda}^{s p}(\mathbf{x}, \overline{\mathbf{x}} ; \mathbf{y}, \overline{\mathbf{y}} \mid \mathbf{a}) & =\prod_{1 \leq i \leq j \leq n}\left(x_{i}+y_{j}+\bar{x}_{i}+\bar{y}_{j}\right) s p_{\mu}(\mathbf{x}, \overline{\mathbf{x}} \mid \mathbf{a}) ; \\
Q_{\lambda}^{s o}(\mathbf{x}, \overline{\mathbf{x}} ; \mathbf{y}, \overline{\mathbf{y}}, 1 \mid \mathbf{a}) & =\prod_{1 \leq i \leq j \leq n}\left(x_{i}+y_{j}+\bar{x}_{i}+\bar{y}_{j}\right) s o_{\mu}(\mathbf{x}, \overline{\mathbf{x}}, 1 \mid \mathbf{a}) .
\end{aligned}
$$

Proof: Again we focus on the symplectic case and start by using (34). By subtracting successive rows as in the proof of Theorem 6 and using 36 with $m=\lambda_{j}-1$ we extract factors $\left(x_{i}+y_{j}+\bar{x}_{i}+\bar{y}_{j}\right)$ to give

$$
\begin{aligned}
& Q_{\lambda}(\mathbf{x}, \overline{\mathbf{x}} ; \mathbf{y}, \overline{\mathbf{y}} \mid \mathbf{a})=\prod_{i=1}^{n}\left(x_{i}+y_{i}+\bar{x}_{i}+\bar{y}_{i}\right)\left|g_{\lambda_{j}-1 ; i, i, n}(\mathbf{x}, \overline{\mathbf{x}} ; \mathbf{y}, \overline{\mathbf{y}} \mid \mathbf{a})\right| \\
& =\prod_{1 \leq i \leq j \leq n}\left(x_{i}+y_{j}+\bar{x}_{i}+\bar{y}_{j}\right)\left|g_{\lambda_{j}-1-n+i ; i, n, n}(\mathbf{x}, \overline{\mathbf{x}} ; \mathbf{y}, \overline{\mathbf{y}} \mid \mathbf{a})\right| \\
& =\prod_{1 \leq i \leq j \leq n}\left(x_{i}+y_{j}+\bar{x}_{i}+\bar{y}_{j}\right)\left|h_{\lambda_{j}-(n-i+1)}\left(\mathbf{x}^{(i)}, \overline{\mathbf{x}}^{(i)} \mid \mathbf{a}\right)\right| \\
& =\prod_{1 \leq i \leq j \leq n}\left(x_{i}+y_{j}+\bar{x}_{i}+\bar{y}_{j}\right)\left|h_{\mu_{j}-j+i}\left(\mathbf{x}^{(i)}, \overline{\mathbf{x}}^{(i)} \mid \mathbf{a}\right)\right|=\prod_{1 \leq i \leq j \leq n}\left(x_{i}+y_{j}+\bar{x}_{i}+\bar{y}_{j}\right) s p_{\mu}(\mathbf{x}, \overline{\mathbf{x}} \mid \mathbf{a}) .(40)
\end{aligned}
$$

Here we have used (35) and the fact that $\lambda=\mu+\delta$ so that $\lambda_{j}=\mu_{j}+n-j+1$ for $j=1,2, \ldots, n$, as well as the symplectic factorial flagged Jacobi-Trudi identity of Theorem 6

\section{Acknowledgements}

This work was supported by the Canadian Tri-Council Research Support Fund. The first author (AMH) acknowledges the support of a Discovery Grant from the Natural Sciences and Engineering Research Council of Canada (NSERC). The second author (RCK) is grateful for the hospitality extended to him while visiting Wilfrid Laurier University, Nankai University and the University of Tokyo, and for the financial support making such visits possible.

\section{References}

[1] L.C. Biedenharn and J.D. Louck, A new class of symmetric polynomials defined in terms of tableaux, Adv. in Appl. Math. 10 (1989), 396-438. 
[2] S. Billey and M. Haiman, Schubert polynomials for the classical groups, J. Amer. Math. Soc. 8 (1995), 443-482.

[3] B. Brubaker, D. Bump and S. Friedberg, Schur polynomials and the Yang-Baxter equation, Comm. in Math. Physics 308 (2011), 1563-1571.

[4] D. Bump, P.J. McNamara and M. Nakasuji, Factorial Schur functions and the Yang-Baxter equation, Comm. Math. Univ. St. Pauli, 63 (2014) 23-45.

[5] W.Y.C. Chen, B. Li and J.D. Louck, The flagged double Schur function, J. Algebraic Comb. 15 (2002), 7-26.

[6] I.P. Goulden and C. Greene, A new tableau representation for supersymmetric Schur functions, J. Algebra 170 (1994), 687-703.

[7] A.M. Hamel and R.C. King, Bijective proofs of shifted tableau and alternating sign matrix identities, J. Algebraic Comb., 25 (2007), 417-458.

[8] A.M. Hamel and R.C. King, Tokuyama's Identity for factorial Schur P and Q functions, Elec. J. Comb. 22.2 (2015), \#P2.42.

[9] T. Ikeda, L.C. Mihalcea and H. Naruse, Double Schubert polynomials for the classical groups, Adv. in Math. 226 (2011), 840-886.

[10] V.N. Ivanov, Combinatorial formula for factorial Schur Q-functions, J. of Math. Sci., 107 (2001), 4195-4211.

[11] R.C. King, Weight multiplicities for the classical Lie groups, in Lecture Notes in Physics, Springer, New York, 150 (1976), 490-499.

[12] I.G. Macdonald, Schur functions: theme and variations, in Séminaire Lotharingien de Combinatoire, Publ. Inst. Rech. Math. Av., Univ. Louis Pasteur, Strasbourg, 498 (1992), 5-39.

[13] S. Okada, Partially strict shifted plane partitions, J. Comb. Th. A 53 (1990), 143-156.

[14] S. Okada, Lattice path methods and characters of classical groups, preprint.

[15] G.I. Olshanski, A. Regev, A. Vershick, Frobenius-Schur functions, arXiv:math/0110077, 2001.

[16] B.E. Sagan, Shifted tableaux, Schur Q-functions and a conjecture of R. Stanley, J. Comb. Th. A 45 (1987), 62-103.

[17] J.R. Stembridge, Nonintersecting paths, pfaffians, and plane partitions, Adv. in Math. 83 (1990), 96-131.

[18] S. Sundaram, Orthogonal tableaux and an insertion algorithm, J. Comb. Th. A 53(1990),239256.

[19] T. Tokuyama, A generating function of strict Gelfand patterns and some formulas on characters of general linear groups, J. Math. Soc. Japan 40 (1988), 671-685.

[20] D.R. Worley, “A Theory of Shifted Young Tableaux,” Ph.D. thesis, MIT, 1984. 